Article

\title{
Super Connectivity of Erdős-Rényi Graphs
}

\author{
Yilun Shang ${ }^{\mathbb{D}}$ \\ Department of Computer and Information Sciences, Faculty of Engineering and Environment, \\ Northumbria University, Newcastle NE1 8ST, UK; yilun.shang@northumbria.ac.uk
}

Received: 9 February 2019; Accepted: 12 March 2019; Published: 15 March 2019

\begin{abstract}
The super connectivity $\kappa^{\prime}(G)$ of a graph $G$ is the minimum cardinality of vertices, if any, whose deletion results in a disconnected graph that contains no isolated vertex. $G$ is said to be $r$-super connected if $\kappa^{\prime}(G) \geq r$. In this note, we establish some asymptotic almost sure results on $r$-super connectedness for classical Erdős-Rényi random graphs as the number of nodes tends to infinity. The known results for $r$-connectedness are extended to $r$-super connectedness by pairing off vertices and estimating the probability of disconnecting the graph that one gets by identifying the two vertices of each pair.
\end{abstract}

Keywords: super connectivity; random graph; interconnection network

\section{Introduction}

The topology of an interconnection network is usually modelled by a simple connected graph $G=(V(G), E(G))$, where $V(G)$ is the vertex set representing the set of processors and $E(G)$ is the set of edges, i.e., communication links in the network. A modern multiprocessor network typically contains tens of thousands of processors, and the reliability and fault-tolerance of such systems are of critical importance, as a local breakdown may cause the fragmentation of the whole network $[1,2]$.

The first graph-theoretic measure of reliability is perhaps the connectivity of $G$, denoted by $\kappa(G)$, which is the minimum number of vertices whose removal results in a disconnected graph or a single vertex. A graph is said to be $k$-connected if $\kappa(G) \geq k$. Evidently, the larger $\kappa(G)$ is, the more reliable the network becomes. The concept of super connectivity was introduced in $[3,4]$ as a more realistic and refined index of reliability as the probability that all processors directly connected to a processor fail at the same time is very small in large-scale communication networks. Specifically, a subset $S \subseteq V(G)$ is called a super vertex-cut if $G \backslash S$ is not connected and every component contains at least two vertices. The super connectivity $\kappa^{\prime}(G)$ is the minimum cardinality over all super vertex-cuts in $G$, if there is any, and is set to be $+\infty$ otherwise. We say that a graph $G$ is $k$-super connected if $\kappa^{\prime}(G) \geq k$. The super connectivity has been investigated extensively for a variety of graphs; see, e.g., [5-10] and the references therein. Super connectivity provides a more realistic measure of reliability for multiprocessor systems since an isolated node has very limited influence on the system performance [4], which also echoes the idea of considering the giant components in network resilience, while ignoring isolated nodes or small components $[2,11]$.

In this letter, we study the super connectivity of two classical Erdős-Rényi random graphs of order $n$, namely the uniform model $G_{n, M}$ and the binomial model $G_{n, p}$ [12]. The graph $G_{n, M}$ can be obtained by starting with an empty graph on $n$ vertices and inserting $M$ edges in such a way that all possible $\left(\begin{array}{c}\left(\begin{array}{l}n \\ 2\end{array}\right) \\ M\end{array}\right)$ choices are equally likely, while the graph $G_{n, p}$ with $p \in[0,1]$ is obtained by including each possible edge with probability $p$ independently of others. Since the introduction of Erdős-Rényi random graphs in the 1950s, they have been developed into an extremely prolific and highly active field in mathematics. We refer the readers to the monographs [12,13] for a variety of results and the recent textbook [11] for some additional variant models in relation to complex networks. The connectivity of 
Erdős-Rényi random graphs has been well understood so far. The following result due to Erdős and Rényi is well known (see [14]; [12], Thm7.7).

Theorem 1 ([14]). Let $M=\frac{n}{2}(\ln n+r \ln \ln n+c), r=0,1,2, \cdots$, and $c \in \mathbb{R}$. Then:

$$
\lim _{n \rightarrow \infty} \mathbb{P}\left(\kappa\left(G_{n, M}\right)=r\right)=1-\exp \left(-\frac{e^{-c}}{r !}\right),
$$

and:

$$
\lim _{n \rightarrow \infty} \mathbb{P}\left(\kappa\left(G_{n, M}\right)=r+1\right)=\exp \left(-\frac{e^{-c}}{r !}\right) .
$$

Basically, in this letter, we manage to derive threshold-like results (see Theorem 2 below) for super connectivity in Erdôs-Rényi random graphs based on Theorem 1. To our knowledge, this is the first attempt to deal with super connectivity in the setting of random graphs. Here, we are interested in the limit of a large graph size, i.e., as $n$ tends to infinity, which is customary in the theory of random graphs.

Remark 1. In [15], a graph $G$ is said to be super connected, or simply super- $\kappa$, if every minimum vertex cut is the neighbourhood of a vertex in $G$, that is every minimum vertex cut isolates a vertex. We clarify that a graph is super-k does not mean that it is one-super connected. A triangle having a dangling vertex attached is a counterexample. On the other hand, a graph is one-super connected does not imply that it is super- $\kappa$ either (consider the bow-tie graph on five vertices).

\section{Main Results}

The following lemma on the existence of perfect matchings in $G_{n, M}$ is useful.

Lemma 1 ([16]; [12], Cor.7.21). If $n$ is even, $\omega(n) \rightarrow \infty$, and $M=\frac{n}{2}(\ln n+\omega(n))$, then:

$$
\lim _{n \rightarrow \infty} \mathbb{P}\left(G_{n, M} \text { has a } 1 \text {-factor }\right)=1 .
$$

Let $\delta(G)$ be the minimum degree of a graph $G$. The property that " $\delta(G)$ is less than a constant" is a monotone graph property. Therefore, the "asymptotic equivalence" (see [13], Cor. 1.16) between the two models $G_{n, M}$ and $G_{n, p}$ for monotone properties and Theorem 3.2 in [11] (or Exercise 3.2 in [12]), which was originally stated for $G_{n, p}$ model, readily give rise to the following estimate.

Lemma 2. Suppose that $k \geq 0$ is an integer, $M=\frac{n}{2}(\ln n+k \ln \ln n+\omega(n))$, where $\omega(n)=o(\ln \ln n)$, as $n \rightarrow \infty$. Then:

$$
\lim _{n \rightarrow \infty} \mathbb{P}\left(\delta\left(G_{n, M}\right) \leq k+1\right)=1 .
$$

Our main result reads as follows.

Theorem 2. Let $r \geq 1$ be an integer, $c \in \mathbb{R}$, and $\omega(n)=o(\ln \ln n)$ as $n \rightarrow \infty$.

(i) If $M \leq \frac{n}{2}\left(\ln n+\left(\left\lceil\frac{r}{2}\right\rceil-2\right) \ln \ln n+\omega(n)\right)$, then:

$$
\lim _{n \rightarrow \infty} \mathbb{P}\left(G_{n, M} \text { is } r \text {-super connected }\right)=0 ;
$$

(ii) If $M \geq \frac{n}{2}(\ln n+r \ln \ln n+c)$, then:

$$
\lim _{n \rightarrow \infty} \mathbb{P}\left(G_{n, M} \text { is } r \text {-super connected }\right)=1 .
$$


Note that $r$-super connectedness is an increasing graph property in the sense that adding edges will never violate the property. It follows immediately from the "asymptotic equivalence" ([13], Cor. 1.16) between the two models $G_{n, M}$ and $G_{n, p}$ that the following corollary holds for the binomial model.

Corollary 1. Let $r \geq 1$ be an integer, $c \in \mathbb{R}$, and $\omega(n)=o(\ln \ln n)$ as $n \rightarrow \infty$.

(i) If $p \leq \frac{1}{n}\left(\ln n+\left(\left\lceil\frac{r}{2}\right\rceil-2\right) \ln \ln n+\omega(n)\right)$, then:

$$
\lim _{n \rightarrow \infty} \mathbb{P}\left(G_{n, p} \text { is r-super connected }\right)=0 ;
$$

(ii) If $p \geq \frac{1}{n}(\ln n+r \ln \ln n+c)$, then:

$$
\lim _{n \rightarrow \infty} \mathbb{P}\left(G_{n, p} \text { is } r \text {-super connected }\right)=1 .
$$

We observe from the above results that there is still a gap, namely $\frac{2 M-n \ln n}{n \ln \ln n} \in\left(\left\lceil\frac{r}{2}\right\rceil-2, r\right)$ for the $G_{n, M}$ model and $\frac{n p-\ln n}{\ln \ln n} \in\left(\left\lceil\frac{r}{2}\right\rceil-2, r\right)$ for the $G_{n, p}$ model, to pinpoint the sharp threshold for $r$-super connectedness. It would be very interesting to determine the asymptotic probability distribution of $r$-super connectedness within these parameter windows (perhaps in a similar flavour as Theorem 1).

Proof of Theorem 2. To show (i), it suffices to show the statement when $M=$ $\frac{n}{2}\left(\ln n+\left(\left\lceil\frac{r}{2}\right\rceil-2\right) \ln \ln n+\omega(n)\right)$. For $r=1,2$, the result follows from the fact that $\mathbb{P}\left(G_{n, M}\right.$ is connected $) \rightarrow 0$ as $n$ goes to infinity (see Theorem 1$)$. In the following, we assume that $r \geq 3$.

First, we assume that $n$ is even. Lemma 1 implies that $G_{n, M}$ has a one-factor asymptotically almost surely (a.a.s.) as $n \rightarrow \infty$. For a given one-factor, we contract $G_{n, M}$ by shrinking each edge in the one-factor to a single vertex and reducing (possible) multiple edges into a single edge. The resulting graph is denoted by $\tilde{G}_{\frac{n}{2}, N}$, which has $\frac{n}{2}$ vertices and $N$ edges. Theorem 1 implies that $G_{n, M}$ is connected a.a.s., and hence, $N \leq \frac{M}{2}$. Indeed, this can be easily seen by noting that the above construction procedure removes at least half of the edges in $G_{n, M}$ (see Figure 1 for an illustration). Thus, $\tilde{G}_{\frac{n}{2}, N}$ can be viewed as a subgraph (in the sense of graph instance) of $G_{\frac{n}{2}, \frac{M}{2}}$.

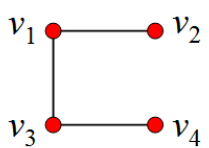

(a)

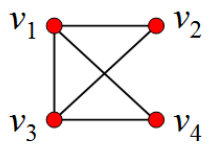

(c)

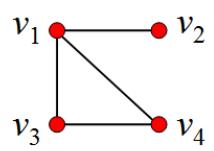

(b)

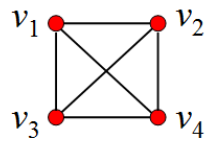

(d)

Figure 1. Four situations of two independent edges $\left\{v_{1}, v_{2}\right\}$ and $\left\{v_{3}, v_{4}\right\}$ in a graph of $n=4$ vertices. The construction process will reduce three edges to one edge for $(\mathbf{a})(3 \rightarrow 1)$. Similarly, $4 \rightarrow 1,5 \rightarrow 1$, and $6 \rightarrow 1$ for $(\mathbf{b}-\mathbf{d})$, respectively. Clearly, (a) is the "worst" case. It is straightforward to verify that, in general, in a graph of $2 k$ vertices, we have $2 k-1 \rightarrow k-1$ for such worst cases, but $\frac{k-1}{2 k-1} \leq \frac{1}{2}$.

By our construction, if $G_{n, M}$ is $r$-super connected, then $\tilde{G}_{\frac{n}{2}, N}$ is $\left\lceil\frac{r}{2}\right\rceil$-connected. Since $r$-super connectedness is an increasing property, it remains to show:

$$
\begin{aligned}
& \lim _{n \rightarrow \infty} \mathbb{P}\left(\kappa\left(G_{\frac{n}{2}, \frac{M}{2}}\right) \geq\left\lceil\frac{r}{2}\right\rceil\right)=0 \text { for } r \geq 3, \\
& \text { if } M=\frac{n}{2}\left(\ln n+\left(\left\lceil\frac{r}{2}\right\rceil-2\right) \ln \ln n+\omega(n)\right) .
\end{aligned}
$$


In view of Lemma 2, we obtain $\lim _{n \rightarrow \infty} \mathbb{P}\left(\delta\left(G_{\frac{n}{2}, \frac{M}{2}}\right) \leq\left\lceil\frac{r}{2}\right\rceil-1\right)=1$. Recall that $\delta(G) \geq \kappa(G)$ holds for any graph $G$. We hence arrive at $\lim _{n \rightarrow \infty} \mathbb{P}\left(\kappa\left(G_{\frac{n}{2}, \frac{M}{2}}\right) \leq\left\lceil\frac{r}{2}\right\rceil-1\right)=1$, which yields (1).

Now if $n$ is odd, the probability that the $n^{\text {th }}$ vertex is isolated turns out to be $o(1)$ (when $M=$ $\left.\frac{n}{2}\left(\ln n+\left(\left\lceil\frac{r}{2}\right\rceil-2\right) \ln \ln n+\omega(n)\right)\right)$. Hence, the above proof strategy still works.

To prove (ii), we note that $\mathbb{P}\left(G_{n, M}\right.$ is $r$-super connected $) \geq \mathbb{P}\left(G_{n, M}\right.$ is $r$-connected $) \rightarrow 1$ as $n \rightarrow$ $\infty$ by using Theorem 1 .

\section{Concluding Remarks}

In this letter, we have obtained some asymptotic almost sure results on the $r$-super connectivity for Erdős-Rényi models $G_{n, M}$ and $G_{n, p}$. A more general concept of conditional connectivity with respect to a graph property $\mathcal{P}$ proposed by Harary [17] is defined as the minimum cardinality of a set of vertices, if any, whose deletion disconnects the graph, and every remaining component has property $\mathcal{P}$. From this viewpoint, the super connectivity here is the special case where $\mathcal{P}$ is the property of having more than two vertices. On the other hand, connectivity properties of varied random graph models (including both relational models, e.g., Erdős-Rényi graphs and geometric models) have been reported recently; see, e.g., [18-21]. It is hoped that this work will stimulate further research effort in these interesting (yet demanding) topics in the realm of random graphs.

Funding: This research received no external funding.

Acknowledgments: The author was supported by a Starting Grant of Northumbria University.

Conflicts of Interest: The author declares no conflict of interest.

\section{References}

1. Albert, R.; Jeong, H.; Barabási, A.-L. Error and attack tolerance of complex networks. Nature 2000, 406, 378-382. [CrossRef] [PubMed]

2. Shang, Y. Vulnerability of networks: Fractional percolation on random graphs. Phys. Rev. E 2014, 89, 012813. [CrossRef] [PubMed]

3. Bauer, D.; Boesch, F.; Suffel, C.; Tindell, R. Connectivity extremal problems and the design of reliable probabilistic networks. In The Theory and Application of Graphs; Wiley: New York, NY, USA, 1981; pp. 45-54.

4. Boesch, F.T. Synthesis of reliable networks: A survey. IEEE Trans. Reliab. 1986, 35, 240-246. [CrossRef]

5. Chang, J.-M.; Chen, X.-R.; Yang, J.-S.; Wu, R.-Y. Locally exchanged twisted cubes: connectivity and super connectivity. Inform. Process. Lett. 2016, 116, 460-466. [CrossRef]

6. Ekinci, G.B.; Gauci, J.B. On the reliability of generalized Petersen graphs. Discrete Appl. Math. 2019, 252, 2-9.

7. Ekinci, G.B.; Kirlangi, A. Super connectivity of Kronecker product of complete bipartite graphs and complete graphs. Discrete Math. 2016, 339, 1950-1953.

8. Lin, C.-K.; Huang, H.-M.; Hsu, L.-H. The super connectivity of the pancake graphs and the super laceability of the star graphs. Theory Comput. Sci. 2005, 339, 257-271. [CrossRef]

9. Yang, M. Super connectivity of balanced hypercubes. Appl. Math. Comput. 2012, 219, 970-975. [CrossRef]

10. Zhu, Q.; Zhang, J.; Li, L.L. The $h$-extra connectivity and $h$-extra conditional diagnosabillity of Bubble-sort star graphs. Discrete Appl. Math. 2018, 251, 322-333. [CrossRef]

11. Frieze, A.; Karoński, M. Introduction to Random Graphs; Cambridge University Press: New York, NY, USA, 2016.

12. Bollobás, B. Random Graphs; Cambridge University Press: New York, NY, USA, 2001.

13. Janson, S.; Łuczak, T.; Rucinski, A. Random Graphs; Wiley: New York, NY, USA, 2000.

14. Erdős, P.; Rényi, A. On the strength of connectedness of a random graph. Acta Math. Acad. Sci. Hungar. 1961, 12, 261-267. [CrossRef]

15. Boesch, F.; Tindell, R. Circulants and their connectivities. J. Graph Theory 1984, 8, 487-499. [CrossRef]

16. Bollobás, B.; Frieze, A. On matchings and hamiltonian cycles in random graphs. Ann. Discrete Math. 1985, $28,23-46$.

17. Harary, F. Conditional connectivity. Networks 1983, 13, 347-357. [CrossRef] 
18. Federico, L.; van der Hofstad, R. Critical window for connectivity in the configuration model. Comb. Prob. Comput. 2017, 26, 660-680. [CrossRef]

19. Fountoulakis, N.; Müller, T. Law of large numbers for the largest component in a hyperbolic model of complex networks. Ann. Appl. Prob. 2018, 28, 607-650. [CrossRef]

20. Iyer, S.K. The random connection model: Connectivity, edge lengths, and degree distributions. Rand. Struct. Algor. 2018, 52, 283-300. [CrossRef]

21. Zhao, J.; Yağan, O.; Gligor, V. On connectivity and robustness in random intersection graphs. IEEE Trans. Autom. Contr. 2017, 62, 2121-2136. [CrossRef]

(C) 2019 by the author. Licensee MDPI, Basel, Switzerland. This article is an open access article distributed under the terms and conditions of the Creative Commons Attribution (CC BY) license (http:/ / creativecommons.org/licenses/by/4.0/). 